\title{
Treatment with adalimumab in a patient with regenerative nodular hyperplasia secondary to azathioprine
}

\author{
Rafael León-Montañes, Claudio Trigo-Salado, Eduardo Leo-Carnerero, \\ María Dolores de-la-Cruz-Ramírez, José Manuel Herrera-Justiniano and José Luis Márquez-Galán
}

Unit of Gastrointestinal Diseases. Hospital Universitario Virgen del Rocío. Seville, Spain

\begin{abstract}
Introduction: regenerative nodular hyperplasia $(\mathrm{RNH})$ is a rare liver disease with an etiology that is not well understood. Among the etiological factors are purine-analogue drugs such as azathioprine.

Case report: we present a case of a 47-year-old patient diagnosed with Crohn's disease in treatment with azathioprine due to corticosteroid dependency who developed $\mathrm{RNH}$ with clinical and laboratory signs of portal hypertension one year after starting treatment. After discontinuation of azathioprine, the patient started treatment and, given the poor disease progression, started treatment with adalimumab. This was continued with an excellent response and without deleterious effects on the liver.

Discussion: the relevance of this case is twofold: First, this is a rare and early side effect of azathioprine treatment and this is an irreversible disease with potentially serious complications. Second, because treatment was carried out with biological drugs (adalimumab) despite the patient having advance liver disease with portal hypertension without any evidence of it worsening, nor signs of deleterious effects or complications, given that there is scarce or no experience with adalimumab treatment in this type of situation.
\end{abstract}

Key words: Nodular regenerative hyperplasia. Adalimumab. Azathioprine.

León-Montañes R, Trigo-Salado C, Leo-Carnerero E, De-la-CruzRamírez MD, Herrera-Justiniano JM, Márquez-Galán JL. Treatment with adalimumab in a patient with regenerative nodular hyperplasia secondary to azathioprine. Rev Esp Enferm Dig 2013;105:168-170.

Received: $18-04-2012$

Accepted: 03-05-2012

Correspondence: Rafael León Montañes. Service of Gastrointestinal Disease. Hospital Universitario Virgen del Rocío. C/ Cardenal Ilundain. 41013 Seville, Spain

e-mail: rafa_leon1@hotmail.com

\section{CASE REPORT}

We present a case of a 47-year-old male, smoker of 10 cigarettes per day, diagnosed with ileocolic Crohn's disease at 20 years of age. The patient has undergone surgery on 5 occasions due to complications of his disease, undergoing broad surgical resections ( $>100 \mathrm{~cm}$ of the terminal ileum in total, ascending colon and sigmoid colon) with a permanent colostomy due to severe stenosing involvement of the distal rectum. Because the patient was corticosteroid dependent, treatment was initiated with azathioprine $100 \mathrm{mg} / 24$ hours, adjusted according to thiopurine S-methyltransferase enzyme activity. Initially there were good results.

At one year from the start of azathioprine treatment, the patient was admitted due to exacerbation of the disease. Laboratory studies revealed slight thrombocytopenia (120,000 platelets), cholestasis, and cytolysis pattern (total bilirubin 2 mg/dl, SGOT 55 IU/l, SGPT 69 IU/l, AP 207 IU/l, GGT 143 IU/l). Abdominal ultrasound and CT scan revealed signs of chronic liver disease with moderate hepatosplenomegaly, increase in the caliber of the portal vein and slight ascites as signs of portal hypertension. Oral endoscopy was performed in order to complete the study, which revealed small esophageal varices.

Afterwards, the azathioprine was discontinued and a liver biopsy was performed via the transjugular approach. This revealed changes consistent with RNH (CD10: + in biliary canaliculi, CD34: + in sinus endothelial cells, Ki76: low proliferative index, $<1-2 \%$, immunohistochemical findings of $\mathrm{RNH}$ ), revealing a liver structure with a nodular configuration with marked regenerative signs and absence of overlying fibrosis. The manometry study performed via the jugular vein revealed a normal hepatic venous pressure gradient (HVPG).

Six months later, scheduled surgery was carried out due to a retroperitoneal abscess of the small intestine with poor disease progress. The ileum was resected $(45 \mathrm{~cm})$ and a 
new intraoperative biopsy revealed a pathology result that coincided with the previous biopsy. Given the short intestine and persistence of the inflammatory activity, treatment was initiated with adalimumab without any complications and with an excellent response after 1 year of follow-up.

\section{DISCUSSION}

Regenerative nodular hyperplasia is a rare liver disease that is defined by diffuse transformation of the liver parenchyma in small nodules (1-3 $\mathrm{mm}$ in diameter), forming areas of tissue hyperplasia alternating with areas of atrophy without septal fibrosis (1-6).

Its etiology and natural history is poorly understood. Cases have been reported secondary to rheumatological diseases (rheumatoid arthritis, Felty's syndrome, SLE, antiphospholipid syndrome, etc.), hematological diseases (idiopathic thrombocytopenic purpura, polycythemia vera, lymphomas, chronic leukemia, hyperhomocysteinemia, etc.), congenital diseases (portal vein agenesis, cardiac anomalies), drugs (azathioprine, 6-thioguanine, busulfan, cyclophosphamide, bleomycin, etc.) and associated with other diseases (celiac disease, tuberculosis, primary biliary cirrhosis, toxic oil syndrome, etc.) (7-15).

Regarding its pathophysiology, it has been suggested that nodular transformation of the liver parenchyma may be secondary to changes in venous blood flow. Morphologically, abnormalities have been seen in the portal vein or in the central vein and many drugs that are associated with RNH also cause other types of vascular lesions. Therefore, reduction in the vascularization of certain areas of the liver parenchyma results in areas on tissue atrophy. To compensate, nodular areas are produced as a hypertrophic response to slightly increased venous flow (1-6).

$\mathrm{RNH}$ is often asymptomatic for years. When it is symptomatic, it causes the clinical signs of portal hypertension (ascites, bleeding due to rupture of esophageal varices, hypersplenism/splenomegaly) (1-6). The manometry study performed in our patient via the jugular vein revealed a normal HVPG.

The gold standard of diagnosis is a liver biopsy that shows areas of parenchymal hyperplasia with the formation of small nodules (1-3 $\mathrm{mm}$ in diameter) without fibrosis alternating with areas of tissue atrophy. Laboratory studies are initially normal. When the disease progresses, there may be a pattern of cytolysis and mild cholestasis (elevation of alkaline phosphatase in $25 \%$ of cases) (1-6).

In our clinical case, the patient was asymptomatic from a liver standpoint, with laboratory tests revealing a mild alteration in liver biochemistry (mild cytolysis and cholestasis) and a moderate thrombocytopenia. Imaging studies (abdominal ultrasound and CT) revealed hepatosplenomegaly and signs of chronic liver disease with ascites, so a liver biopsy was performed and was ultimately diagnostic.

The risk of RNH is patients treated with azathioprine is $0.50 \%$ at 5 years from the start of treatment and $1.25 \%$ at
10 years, though its incidence may be underestimated given its initially asymptomatic course and the absence of a specific diagnostic laboratory or imaging test $(1,2)$.

The duration from the start of azathioprine treatment and the diagnosis of $\mathrm{RNH}$ varies between studies, being around 50 months (some cases are described from 6 months of starting treatment). In our patient, 12 months passed form the introduction of the treatment and the diagnosis of $\mathrm{RNH}(1,2)$. Our patient had all of the risk factors that are currently associated with RNH in patients with Crohn's disease on azathioprine treatment; male gender, stenosing behaviour, bowel resection more than $50 \mathrm{~cm}$, and with less power of association, ileal location and tobacco use (1-4). Other studies describe an increase in the incidence of $\mathrm{RNH}$ in patients with ulcerative colitis versus patients with Crohn's disease (15).

$\mathrm{RNH}$ is an irreversible disease and its progression is still not well understood, though its prognosis and subsequent progression is more favorable with resolution of the underlying cause. Its natural evolution is marked by the development of portal hypertension and its complications. Control of $\mathrm{pH}$ is the basis of therapeutic management in these patients. On successive revisions, in addition to endoscopic monitoring to evaluate signs of portal hypertension (primarily esophageal varices), screening is recommended for liver carcinoma using abdominal ultrasound and alpha-fetoprotein, as there is an increase in liver carcinogenesis over the course of RNH (1-6).

There is little experience with biological treatment in patients with RNH. Given the patient's situation of inflammatory activity following the diagnosis of $\mathrm{RNH}$, and despite having signs of portal hypertension (esophageal varices, ascites, hypersplenism/splenomegaly), treatment was started with adalimumab in order to induce remission of the Crohn's disease and to maintain remission. The patient had an excellent response and did not have any complications. There were no changes in liver function tests or radiological studies (ultrasound and CT), nor were there any changes on endoscopy after one year of follow-up.

\section{REFERENCES}

1. Selsik P, Mary JY, Beaugerie L, Lémann M, Colombel JF, VernierMassouille $\mathrm{G}$, et al. Incidence of nodular regenerative hyperplasia in inflammatory bowel disease patients treated with azathioprine. Inflam Bowel Dis 2011;17:565-72.

2. Vernier-Massouille G, Cosnes J, Lemann M, Marteau P, Reinisch W, Laharie D, et al. Nodular regenerative hyperplasia in patients with inflammatory bowel disease treated with azathioprine. Gut 2007; 56:1404-9.

3. Lopez Martín C, De la Fuente Fernández E, Corbatón P, Sánchez MC, Gisbert JP. Nodular regenerative hyperplasia: Azathiprine induced hepatoxicity in a patients with Crohn s disease. Gastroenterol and Hepatol 2011;34:16-9.

4. Calabrese E, Hanauer SB. Assesment of non-cirrhotic portal hypertension associated with thiopurine therapy in inflammatory bowel disease. J Crohns Colitis 2011;5:48-53.

5. Ferlitsch A, Teml A, Reinisch W, Ulbrich G, Wrba F, Homoncik M, et al. 6-Thioguanine associated nodular regenerative hyperplasia in patients with inflammatory Bowel disease may induce portal hypertension. Am J Gastroenterol 2007;102:2495-503. 
6. Reshamwala PA, Kleiner DE, Heller T. Nodular regenerative hyperplasia: Not all nodules are created equal. Hepatology 2006;44:7-14.

7. O Buchel, T Roskams, Van Damme B, Nevens F, Pirenne J, Fevery J. Nodular regenerative hyperplasia, portal vein thrombosis, and avascular hip necrosis due to hyperhomocysteinaemia Gut 2005;54:1021-3.

8. Wanless IR. Micronodular transformation (nodular regenerative hyperplasia) of the liver: A report of 64 cases among 2,500 autopsies and a new classification of benign hepatocellular nodules. Hepatology 1990;11:787-97.

9. Austin A, Campbell E, Lane P, Elias E. Nodular regenerative hyperplasia of the liver and coeliac disease: Potential role of IgA anticardiolipin antibody. Gut 2004;53:1032-4.

10. Arnott ID, Ghosh S. Portal hypertension in the presence of minimal liver damage in Crohn's disease on long-term azathioprine: Possible endothelial cell injury. Eur J Gastroenterol Hepatol 2000;12:569-73.
11. Daniel F, Cadranel JF, Seksik P, Cazier A, Duong Van Huyen JP, Ziol $\mathrm{M}$, et al. Azathioprine induced nodular regenerative hyperplasia in IBD patients. Gastroenterol Clin Biol 2005;29:600-3.

12. Steiner PE. Nodular regenerative hyperplasia of the liver. Am J Pathol 1959;35:943-53.

13. Grazioli L, Alberti D, Olivetti L, Rigamonti W, Codazzi F, Matricardi $\mathrm{L}$, et al. Congenital absence of portal vein with nodular regenerative hyperplasia of the liver. Eur Radiol 2000;10:820-5.

14. Trenschel GM, Schubert A, Dries V, Benz-Bohm G. Nodular regenerative hyperplasia of the liver: Case report of a 13-year-old girl and review of the literature. Pediatr Radiol 2000;30:64-8.

15. Martínez F, Nos P, Pastor M, Garrigues V, Ponce J. Adverse effects of azathioprine in the treatment of inflammatory bowel disease. Rev Esp Enferm Dig 2001;93:769-78. 